\title{
Rational trust
}

\author{
Nikolaj Jang Lee Linding Pedersen • Kristoffer Ahlström-Vij • \\ Klemens Kappel
}

Received: 23 March 2014 / Accepted: 23 March 2014 / Published online: 5 April 2014

(C) Springer Science+Business Media Dordrecht 2014

Trust plays a crucial role in our cognitive and practical lives. We exhibit an attitude of trust towards others as sources of information by acquiring beliefs through their testimony and by acting on them. We trust others to do certain things or display a certain kind of behaviour.

Trust has a normative dimension: it can be rational or not. The five articles in this special issue explore this normative dimension. Karen Frost-Arnold starts her article "The cognitive attitude of rational trust" with the observation that trust can be underwritten by a variety of reasons. One type of reason is epistemic: one might trust someone to speak truly because of evidence of her reliability. Other types of reason are therapeutic, coping, or corrective in nature. One might trust someone's testimony in order to inspire a higher degree of honesty in that person, to cope better with certain cognitive tasks, or to correct a bias or prejudice that one holds against the person. When underwritten by epistemic reasons, it seems reasonable to take the attitude constitutive of trust to be belief. However, when underwritten by the other three types of reason, belief does not seem like the right kind of attitude. Belief is involuntary, controlled by evidence. But therapeutic, coping, and corrective trust can be placed voluntarily-in a way not regulated by evidence. It has proved difficult to find a single analysis that can adequately accommodate all of these different types of trust. Frost-Arnold's aim is to provide such an account. Practical reasoning plays a crucial role in her account: $A$ 's

\footnotetext{
N. J. L. L. Pedersen ( $ه)$

Underwood International College, Yonsei University, Seodaemun-gu, Seoul, South Korea e-mail: nikolaj@yonsei.ac.kr

K. Ahlström-Vij

University of Kent, Canterbury, United Kingdom

K. Kappel

University of Copenhagen, Copenhagen, Denmark
} 
trusting $B$ to $\varphi$ amounts to $A$ 's believing or accepting that $B$ will $\varphi$, and this belief or acceptance being taken as a premise in $A$ 's practical reasoning.

In "The practical rationality of trust" Paul Faulkner likewise seeks to offer an account of what it is for $A$ to trust $B$ to $\varphi$. Faulkner's account is developed against the background of a criticism of Humean accounts, according to which acts of trusting are explained in terms of beliefs and desires. This kind of account is teleological in nature because it explains action by reference to a desire to reach some end. Faulkner argues that the Humean or teleological approach is not adequate. Adequate accounts of acts of trusting must classify the trusted-the person trusted to $\varphi$-as following a norm of trust. However, on the Humean view it is only possible to classify the trusted as conforming to such a norm. Having argued this critical point, Faulkner develops a positive account. According to Faulkner, the trusted must see the other person's trust as a reason to $\varphi$. It is crucial that this dependence is seen as a reason and motivates the trusted in acting. This puts the idea of arche at the heart of the proposed account. The source of the trusted's act of $\varphi$-ing must be considered. The trusted must act out of trust.

Aron Vallinder and Erik Olsson's “Trust and the value of overconfidence: a Bayesian perspective on social network communication" is framed within Laputa, a computer model of social networks developed by Staffan Angere. Social networks consist of enquirers linked via communication channels, enabling them to send and receive information. Enquirers are assumed to investigate the question whether $p$. At any given time, each enquirer assigns a credence (subjective probability) to $p$. Also, at any given time, each enquirer assigns a specific degree of trust to information sources in the network (other enquirers as well as instruments, data bases, and other sources of information). In the first part of the paper Vallinder and Olsson discuss two connected questions that arise naturally when considering information flow in social networks: (i) how should an enquirer update her credence (subjective probability) in $p$ in light of new information, and (ii) how should an enquirer update her trust in a source from which she receives information? Laputa is a Bayesian model, and accordingly, Vallinder and Olsson give question (i) a Bayesian answer: enquirers should update their credences by conditionalizing on the information received. To address (ii) Vallinder and Olsson first develop an account of trust within Laputa. They treat an enquirer's trust in a given source as the enquirer's credence in the reliability of that source and then address (ii) by specifying a Bayesian update rule. In the second part of the paper, Vallinder and Olsson discuss overconfidence. In Laputa, an enquirer $E$ 's being overconfident about a source $S$ amounts to $E$ 's credence in the reliability of $S$ being higher than $S$ 's actual reliability. Interestingly, in the Laputa model, enquirers with a relatively low degree of reliability benefit epistemically from overestimating their own reliability, but they rarely stand to gain anything from an epistemic point of view by overestimating the reliability of others. Vallinder and Olsson offer an explanation of this interesting difference.

Klemens Kappel's contribution-“Believing on trust"-is centered around certain cases of division of cognitive labour. In the target cases no single individual is capable of executing the entire project on her own. The division of cognitive labour is thus not merely a practical arrangement that enables one or more members of the group to execute a project that they could in principle execute on their own. Rather, it is 
needed in order to execute the project in the first place. According to John Hardwig, in such cases, it is only possible for individual members of the group to know or be justified in believing the deliverances of the group's joint efforts provided that they trust the other group members. This is because individual members are not in a position to independently verify or check the parts of the project for which they are not responsible. Instead they have to trust the relevant group members. Having discussed various ideas in Hardwig's work, Kappel presents his own account of trust. According to Kappel, a subject $S_{1}$ trusts a subject $S_{2}$ with respect to a particular domain of enquiry $D$ if and only if $S_{1}$ has a non-doxastic disposition to accept as true what $S_{2}$ says about $D$. As defined, trust is not by itself sufficient to underwrite knowledge or justified belief. The disposition must be discriminating and defeater-sensitive. Subjects who are disposed to believe the deliverances of any source whatsoever do not acquire knowledge or justified belief even in cases where the source trusted is in fact reliable, as they fail to discriminate between reliable and unreliable sources. Similarly, subjects who are not sensitive to defeaters - e.g. a cue indicating that the trusted source is unreliable-fail to acquire knowledge or justified belief. One might wonder what, ultimately, makes discriminating, defeater-sensitive trust capable of underwriting justified belief and knowledge. Kappel gives a reliabilist answer: forming beliefs through discriminating, defeater-sensitive trust is a reliable way of forming beliefs.

The last contribution - "Partiality and prejudice in trusting" by Katherine Hawleyfocuses on trust and friendship. Trust is subject to both demands of epistemic rationality and demands of friendship. Hawley's aim is to discuss how these two kinds of demands interact. Suppose that it is epistemically permissible for $S$ to trust a friend of hers, $F$, because $S$ 's evidence neither epistemically requires her to believe $F$ trustworthy nor requires her to believe $F$ untrustworthy. In that case $S$ would run a risk by trusting $F$. However, according to Hawley, the fact of friendship obligates $S$ to run the risk of trusting $F$. How about situations where $S$ is epistemically required to believe that $F$ is untrustworthy due to strong evidence or where $S$ is epistemically required not to believe $F$ trustworthy due to a lack of sufficient evidence? In situations of this kind it would seem that there is a tension between the demands of epistemic rationality and the demands of friendship. The former rules against $S$ 's trusting $F$, while the latter seems to provide a reason for trust. This is a view held by Sarah Stroud and Simon Keller. Hawley claims that the tension is only apparent. She offers a two-part argument to support this claim. Hawley argues, first, that epistemic rationality may be more permissible than Stroud and Keller think, and, second, that friendship may be less demanding. Combining these two parts it is more difficult for the demands of epistemic rationality and the demands of friendship to come into tension. 